WORKING GROUP FOR PLANETARY SYSTEM NOMENCLATURE (WGPSN)

(GROUP DE TRAVAIL POUR LA NOMENCLATURE DU SYSTEM PLANETAIRE)

(Committee of the Executive Committee)

PRESIDENT: K. Aksnes

MEMBERS: $\quad$ R. Batson, A. Brahic, M. Fulchignoni, M.Ya. Marov

D. Morrison, T.C. Owen, V.V. Shevchenko, B.A. Smith

L.A. Soderblom

CONSULTANTS: J.M. Boyce, P. Masson

We are sad to report that WGPSN's first President, Dr. Peter Millman, passed away on December 11, 1990 at the age of 84 . He was a world authority on meteors, and a leading figure in Canadian astronomy for many years. Dr. Millman was a very dedicated leader of WGPSN from its inception in 1973 until 1982.

WGPSN held its 19th meeting in Florence, Italy 29-30 April 1991 and the 20th meeting in Buenos Aires, Argentina $30 \mathrm{July} 1991$ during the IAU General Assembly. Much work is also being done by correspondance, increasingly by electronic mail.

Names were recommended for one satellite of Saturn and for six satellites, three rings, and three ring arcs of Neptune. These objects had all been discovered in Voyager mission photographs. The success of the Magellan mission in mapping Venus' surface in great detail has created a need for many new Venus names of which 121 are introduced below. Also 79 new Mars features have been named; they will appear on a new Mars map needed for the Mars Observer mission in a few years time.

The working Group is asking for IAU approval of the following new resolutions:

1. In proposing names for newly discovered satellites of the Solar System, it is recommended that first consideration be given to procedures already established within the IAU, and that confusing duplication with asteroids be avoided. (Amendment of Res. 1 in IAU Trans. XXX, 1988).

2. Three new feature categories are introduced for Venus:

RETICULUM, RETICULA reticular (netlike) pattern FARRUM, FARRA

VALLIS, VALLES row of pancake-like structures sinuous valley

The following names, and those listed in Reports on Astronomy, Vol. XXIA, 613-619,1991, have the approval of the IAU Executive Committee (since August 1991): 
MARS

Name

CRATER

Ayacucho

Bentham

Cangwu

Charlieu

Chatturat

Dunhuang

Dzeng

Ellsley

Escorial

Fenagh

Gandzani

Handlova

Irharen

Jampur

Kamativi

Kamloops

Kisambo

Krasnoye

Leleque

Lodwar

Lomela

Lowbury

Mari

Mendota

Milford

Nipigon

Ocampo

oodnadatta

Rayadurg

Reykholt

Sarn

Suata

Woolgar

CATENA

Apodis Catena

Baphyras Catena

CAVUS

Argyre Cavi

Octantis Cavi

CHAOS

Aurorae Chaos
Lat Long

$38.5 \mathrm{~N}$

$56.0 \mathrm{~S}$

$42.1 \mathrm{~N}$

$38.3 \mathrm{~N}$

$35.7 \mathrm{~N}$

$80.9 \mathrm{~S}$

$80.5 \mathrm{~S}$

$36.5 \mathrm{~N}$

$77.0 \mathrm{~N}$

$34.6 \mathrm{~N}$

$34.5 \mathrm{~N}$

$37.9 \mathrm{~N}$

$34.8 \mathrm{~N}$

$38.8 \mathrm{~N}$

$20.7 \mathrm{~S}$

$53.9 \mathrm{~S}$

$34.3 \mathrm{~N}$

$36.1 \mathrm{~N}$

$36.7 \mathrm{~N}$

$55.4 \mathrm{~S}$

$81.7 \mathrm{~S}$

$42.8 \mathrm{~N}$

$52.4 \mathrm{~S}$

$36.1 \mathrm{~N}$

$52.6 \mathrm{~S}$

$34.0 \mathrm{~N}$

$32.9 \mathrm{~N}$

$52.7 \mathrm{~S}$

$18.6 \mathrm{~S}$

$40.8 \mathrm{~N}$

$77.5 \mathrm{~S}$

$19.2 \mathrm{~S}$

$34.8 \mathrm{~N}$

27.25

$38.8 \mathrm{~N}$

$256.8 \mathrm{~W}$

$84.0 \mathrm{~W}$

$49.1 \mathrm{~S}$

$52.7 \mathrm{~S}$

$40.2 \mathrm{~W}$

$45.6 \mathrm{~W}$

$80.6 \mathrm{~S}$

92. OW

40. $3 \mathrm{~W}$

$89.5 \mathrm{~W}$

$84.1 \mathrm{~W}$

$94.8 \mathrm{~W}$

$48.7 \mathrm{~W}$

$70.5 \mathrm{~W}$

$83.0 \mathrm{~W}$

$54.3 \mathrm{~W}$

$215.7 \mathrm{~W}$

$90.8 \mathrm{~W}$

$88.4 \mathrm{~W}$

$219.2 \mathrm{~W}$

$81.0 \mathrm{~W}$

$260.0 \mathrm{~W}$

$32.1 \mathrm{~W}$

$89.0 \mathrm{~W}$

$216.2 \mathrm{~W}$

$221.9 \mathrm{~W}$

$43.0 \mathrm{~W}$

$56.0 \mathrm{~W}$

93. $\mathrm{W}$

45. $7 \mathrm{~W}$

$221.8 \mathrm{~W}$

$45.1 \mathrm{~W}$

$81.9 \mathrm{~W}$

$221.7 \mathrm{~W}$

$34.8 \mathrm{~W}$

$257.6 \mathrm{~W}$

$85.8 \mathrm{~W}$

$54.5 \mathrm{~W}$

$253.3 \mathrm{~W}$

$85.5 \mathrm{~W}$

$34.5 W$
Town in Bolivia

Town in England

Town in China

Town in France

Town in Thailand

Town in China

Town in Cameroon

Town in England

Town in Spain

Town in Ireland

Town in Georgian SSR

Town in Czechoslovakia

Town in Algeria

Town in Pakistan

Town in Zimbabwe

Town in Canada

Town in Zaire

Town in Russia

Town in Argentina

Town in Kenya

Town in Zaire

Town in New Zealand

Ruined city in Syria

Town in USA

Town in Utah, USA

Town in Canada

Town in Mexico

Town in Australia

Town in India

Town in Iceland

Town in wales

Town in Venezuela

Town in Australia

Classical albedo name

Classical river

Albedo name

Albedo name

Albedo name 
MARS (Cont.)

Name

Lat Long

$7.9 \mathrm{~S}$

$119.4 \mathrm{~W}$

$8.7 \mathrm{~N} \quad 105.5 \mathrm{~W}$

$3.8 \mathrm{~N} \quad 111.1 \mathrm{~W}$

$33.9 \mathrm{~N}$

$82.7 \mathrm{~W}$

DORSUM

Atrax Dorsum

Auxo Dorsum

Charis Dorsum

Cleia Dorsum

Hegemone Dorsum

Pasithea Dorsum

Phaenna Dorsum

FOSSA

Chalce Fossa

Mangala Fossa

Pavonis Fossae

Tanais Fossae

Tyrrhena Fossae

MENSA

Ascraeus Mensa

MONS

Chalce Montes

Galaxius Mons

Gonnus Mons

Horarum Mons

Octantis Mons

Pindus Mons

Tanaica Montes

Tyrrhena Mons

PATERA

Angusta Patera

Australis Patera

Issedon Paterae

PLANUM

Ascuris Planum
$56.1 \mathrm{~S} \quad 41.9 \mathrm{~W}$

$55.8 \mathrm{~S} \quad 41.2 \mathrm{~W}$

$55.3 \mathrm{~S} \quad 46.3 \mathrm{~W}$

$55.3 \mathrm{~S} \quad 44.9 \mathrm{~W}$

$55.7 \mathrm{~S} \quad 41.8 \mathrm{~W}$

$54.3 \mathrm{~S} \quad 43.2 \mathrm{~W}$

51.95

$16.5 \mathrm{~S}$

$4.2 \mathrm{~N}$

$38.6 \mathrm{~N}$

$22.1 \mathrm{~S}$

$11.7 \mathrm{~N}$

$107.8 \mathrm{~W}$

$53.8 \mathrm{~S}$

$35.0 \mathrm{~N}$

$41.6 \mathrm{~N}$

$51.3 \mathrm{~S}$

$55.5 \mathrm{~S}$

$39.7 \mathrm{~N}$

$39.7 \mathrm{~N}$

$24.5 \mathrm{~S}$

$40.0 \mathrm{~W}$

$148.8 \mathrm{~W}$

$111.2 \mathrm{~W}$

$85.3 \mathrm{~W}$

$254.5 \mathrm{~W}$

$37.0 \mathrm{w}$

$217.7 \mathrm{~W}$

$90.8 \mathrm{~W}$

$36.4 \mathrm{~W}$

$42.5 \mathrm{~W}$

$88.9 \mathrm{~W}$

$90.8 \mathrm{~W}$

$258.7 \mathrm{~W}$

$80.7 \mathrm{~S}$

$79.5 \mathrm{~W}$

$51.5 \mathrm{~W}$

$89.9 \mathrm{~W}$

$38.8 \mathrm{~N}$

$39.5 \mathrm{~N}$

84. $0 \mathrm{~W}$
$38.3 \mathrm{~N} \quad 89.1 \mathrm{~W}$
Classical albedo name

Attribute

Albedo name

Classical albedo name

Albedo name

Classical town

One of the Graces

One of the Graces

One of the Graces

One of the Graces

One of the Graces

One of the Graces
Classical albedo name

\author{
Albedo name \\ Named for nearby vallis \\ Albedo name \\ Classical albedo name \\ Classical albedo name
}

Classical albedo name

Albedo name

Classical albedo name

Classical town

Albedo name

Albedo name

Mountains near Vale of Tempe

Classical albedo name

Classical albedo name

Albedo name

Albedo name

Classical albedo name 
MARS(Cont. )

Name

Lat Long

Attribute

SULCUS

Arsia Sulci

\section{$6.4 \mathrm{~S}$}

$129.7 \mathrm{~W}$

Ascraeus Sulci

$11.9 \mathrm{~N} \quad 108.7 \mathrm{~W}$

Pavonis Sulci

$3.9 \mathrm{~N} \quad 117.6 \mathrm{~W}$

Albedo name
Classical albedo name
Albedo name

THOLUS

Issedon Tholus

$36.3 \mathrm{~N}$

$94.6 \mathrm{~W}$

$36.1 \mathrm{~N}$

$85.0 \mathrm{~W}$

E. Mareotis Tholus

$36.8 \mathrm{~N}$

$86.0 \mathrm{~W}$

w. Mareotis Tholus

$35.8 \mathrm{~N}$

$87.5 \mathrm{~W}$

\section{VALLES}

Dzigai Vallis

Enipeus Vallis

$59.7 \mathrm{~S}$

$31.3 W$

$37.5 \mathrm{~N}$

$93.1 \mathrm{~W}$

Nia Vallis

$54.3 \mathrm{~S}$

$33.0 \mathrm{~W}$

Pallacopas Vallis

$54.5 \mathrm{~S}$

$21.2 \mathrm{~W}$

Surius Vallis

60.35

$51.0 \mathrm{~W}$

Classical albedo name

Classical albedo name

classical albedo name

Classical albedo name

\section{VENUS}

\begin{tabular}{lrrrl} 
& \multicolumn{2}{c}{ VENUS } & & \\
Name & Lat & Long & $\begin{array}{l}\text { Diam } \\
(\mathrm{km})\end{array}$ \\
CRATER & & & & \\
Adivar & $8.9 \mathrm{~N}$ & $75.9 \mathrm{E}$ & 30 & Turkish educator \\
Aglaonice & $26.5 \mathrm{~S}$ & $339.9 \mathrm{E}$ & 66 & Ancient astronomer \\
Agnesi & $39.5 \mathrm{~S}$ & $37.8 \mathrm{E}$ & 40 & Italian mathematician \\
Agrippina & $33.2 \mathrm{~S}$ & $65.2 \mathrm{E}$ & 37 & Roman empress \\
Alcott & $59.5 \mathrm{~S}$ & $354.5 \mathrm{E}$ & 71 & American author \\
Al-Taymuriyya & $32.9 \mathrm{~N}$ & $336.2 \mathrm{E}$ & 22 & Egyptian author \\
Amalasthuna & $11.5 \mathrm{~S}$ & $342.4 \mathrm{E}$ & 18 & Ostrogoth queen \\
Amaya & $11.3 \mathrm{~N}$ & $89.1 \mathrm{E}$ & 32 & Spanish Gypsy dancer \\
Amenardes & $15.0 \mathrm{~N}$ & $54.1 \mathrm{E}$ & 25 & Egyptian princess \\
Andami & $17.5 \mathrm{~S}$ & $26.3 \mathrm{E}$ & 28 & Iranian doctor \\
Anicia & $26.4 \mathrm{~S}$ & $31.1 \mathrm{E}$ & 30 & Greek physician and poet \\
Annia Faustina & $22.1 \mathrm{~N}$ & $4.6 \mathrm{E}$ & 20 & Roman empress \\
Astrid & $21.4 \mathrm{~S}$ & $335.5 \mathrm{E}$ & 12 & Scandinavian first name \\
Aurelia & $20.3 \mathrm{~N}$ & $331.8 \mathrm{E}$ & 31 & Julius Caesar's mother \\
Avviyar & $18.0 \mathrm{~S}$ & $353.6 \mathrm{E}$ & 21 & Tamil poet \\
Badarzewska & $22.6 \mathrm{~S}$ & $137.0 \mathrm{E}$ & 28 & Polish composer \\
Ban zhao & $17.2 \mathrm{~N}$ & $146.9 \mathrm{E}$ & 38 & Chinese historian \\
Barrera & $16.6 \mathrm{~N}$ & $109.3 \mathrm{E}$ & 25 & 16th Cen. medical writer \\
Barton & $27.4 \mathrm{~N}$ & $337.5 \mathrm{E}$ & 50 & American Red Cross founder \\
Bassi & $19.0 \mathrm{~S}$ & $64.6 \mathrm{E}$ & 35 & Italian physicist \\
Behn & $32.5 \mathrm{~S}$ & $141.8 \mathrm{E}$ & 25 & English writer \\
Bergolts & $28.1 \mathrm{~S}$ & $80.8 \mathrm{E}$ & 30 & Russian poet \\
& & & &
\end{tabular}

Valley in Navajo Classical river Lowell canal name Lowell canal name Lowell canal name 
VENUS (Cont.)

Name

CRATER

Blixen

Bonnevie

Boulanger

Bourke-White

Bridgit

Buck

Budevska

Callas

Callirhoe

Carreno

Carson

Chapelle

Chiyojo

Cori

Cunitz

Cynthia

Danilova

De Beauvoir

De Lalande

Deloria

Devorguilla

Erxleben

Ferber

Ferrier

Festa

Flagstad

Frank

Fredegonde

Germain

Goppert-Mayer

Greenaway

Guilbert

Halle

Hellman

Henie

Hepworth

Himiko

Holiday

Horner

Hua Mulan

Huang Daopo

Hwangcini

Joliot-Curie

Kartini

Kollwitz
Lat Long Diam Attribute

$59.9 \mathrm{~S}$

$36.1 \mathrm{~S}$

$26.5 \mathrm{~S}$

$21.2 \mathrm{~N}$

45.35

$5.7 \mathrm{~S}$

$0.5 \mathrm{~N}$

$2.4 \mathrm{~N}$

$21.3 \mathrm{~N}$

$3.9 \mathrm{~S}$

$24.2 \mathrm{~s}$

$6.4 \mathrm{~N}$

$47.8 \mathrm{~S}$

$25.4 \mathrm{~N}$

$14.5 \mathrm{~N}$

$16.7 \mathrm{~s}$

$26.4 \mathrm{~S}$

$2.0 \mathrm{~N}$

$20.3 \mathrm{~N}$

$32.0 \mathrm{~S}$

$15.3 \mathrm{~N}$

$50.9 \mathrm{~s}$

$26.4 \mathrm{~N}$

$15.8 \mathrm{~N}$

$11.5 \mathrm{~N}$

$54.3 \mathrm{~S}$

$13.2 \mathrm{~s}$

$50.7 \mathrm{~s}$

$38.0 \mathrm{~S}$

$59.8 \mathrm{~N}$

$22.9 \mathrm{~N}$

$57.9 \mathrm{~S}$

$19.8 \mathrm{~S}$

$4.8 \mathrm{~N}$

$52.0 \mathrm{~S}$

$5.2 \mathrm{~N}$

$19.0 \mathrm{~N}$

$46.7 \mathrm{~s}$

$23.4 \mathrm{~N}$

$86.8 \mathrm{~N}$

$54.2 \mathrm{~S}$

$6.3 \mathrm{~N}$

$1.6 \mathrm{~S}$

$57.8 \mathrm{~N}$

$25.2 \mathrm{~N}$

( km)

$347.5 \mathrm{E}$

$337.3 \mathrm{E}$

96. $0 \mathrm{E}$

$354.9 \mathrm{E}$

$97.0 \mathrm{E}$

$3.8 \mathrm{E}$

39. $3 \mathrm{E}$

$13.0 \mathrm{E}$

$111.1 \mathrm{E}$

27. $2 \mathrm{E}$

$18.9 \mathrm{E}$

$12.9 \mathrm{E}$

$92.9 \mathrm{E}$

63.5E

26.5E

$145.1 \mathrm{E}$

$13.3 \mathrm{E}$

$145.4 \mathrm{E}$

$356.2 \mathrm{E}$

$145.8 \mathrm{E}$

$94.7 \mathrm{E}$

$124.2 \mathrm{E}$

$12.7 \mathrm{E}$

$97.5 \mathrm{E}$

$337.7 \mathrm{E}$

165.1E

$141.7 \mathrm{E}$

$62.1 \mathrm{E}$

333.0E

$133.6 \mathrm{E}$
22 Danish writer

85 Norwegian biologist

57 French composer

31 American photo-journalist

11 First name from Ireland

22 American writer

20 Bulgarian princess

30 Greek opera singer

32 Greek sculptor

57 Venezuelan musician

41 American biologist, author

23 American photo-journalist

35 Japanese (Edo) poetess

50 Czech biochemist

48 Polish astronomer

19 Greek first name
50 Russian ballet dancer

40 French writer

20 French astronomer

38 American anthropologist

22 Irish heroine

28 German scholar

23 American author

30 English singer

25 Italian painter

48 Norwegian opera singer

20 Dutch author

26 Frankish queen

33 French mathematician

35 U.S. physicist(Polish-born)

85 English author, artist

30 French singer

23 Austrian violonist

24 American playwright

70 Norwegian figure scater

54 English sculptoress

35 Japanese queen

24 American singer

28 19th Cen. naturalist

23 Chinese warrior

27 Chinese engineer

30 16th Cen. Korean poet

80 French physicist

24 Javnese educator

30 German artist 
VENUS (Cont.)

\begin{tabular}{|c|c|c|c|c|}
\hline Name & Lat & Long & Diam & Attribute \\
\hline CRATER & & & $(\mathrm{km})$ & \\
\hline Kushinada & $5.7 \mathrm{~S}$ & $76.6 \mathrm{E}$ & 23 & Japanese (Izumo) poetess \\
\hline Lachappelle & $26.7 \mathrm{~N}$ & $336.5 \mathrm{E}$ & 35 & French researcher \\
\hline Landovska & $84.5 \mathrm{~N}$ & $74.2 \mathrm{E}$ & 33 & Polish instrumentalist \\
\hline Lehmann & $44.1 \mathrm{~S}$ & $38.7 \mathrm{E}$ & 20 & Danish geophysicist \\
\hline Li Qingzhao & $23.7 \mathrm{~N}$ & $94.3 \mathrm{E}$ & 21 & Chinese poetess \\
\hline Lullin & $23.1 \mathrm{~N}$ & $81.0 \mathrm{E}$ & 24 & Swiss entomologist \\
\hline Manzolini & $25.7 \mathrm{~N}$ & $91.1 \mathrm{E}$ & 42 & Italian anatomist, teacher \\
\hline Maria Celeste & $23.5 \mathrm{~N}$ & $140.5 \mathrm{E}$ & 90 & Galileo's daughter(Ital.) \\
\hline Marsh & $63.7 \mathrm{~S}$ & $46.7 \mathrm{E}$ & 35 & New Zealand writer \\
\hline Mead & $12.5 \mathrm{~N}$ & $57.4 \mathrm{E}$ & 280 & American anthropologist \\
\hline Merian & $34.5 \mathrm{~N}$ & $76.2 \mathrm{E}$ & 20 & Dutch entomologist \\
\hline Millay & $24.4 \mathrm{~N}$ & $110.9 \mathrm{E}$ & 45 & American poet \\
\hline Monna Lisa & $25.6 \mathrm{~N}$ & $25.3 \mathrm{E}$ & 80 & Ital. model for L. da Vinci \\
\hline Mu Guiying & $41.2 \mathrm{~N}$ & $80.7 \mathrm{E}$ & 25 & Chinese warrior \\
\hline Nemcova & $5.9 \mathrm{~N}$ & 125. OE & 24 & Czech novelist, poet \\
\hline Nijinskaya & $25.9 \mathrm{~N}$ & $122.3 \mathrm{E}$ & 30 & Russian dancer \\
\hline O'Connor & $26.0 \mathrm{~s}$ & $143.8 \mathrm{E}$ & 27 & American novelist \\
\hline Parra & $20.5 \mathrm{~N}$ & $78.1 \mathrm{E}$ & 50 & Chilean writer \\
\hline Piaf & $0.8 \mathrm{~N}$ & $5.1 \mathrm{E}$ & 30 & French singer \\
\hline Recamier & $12.5 \mathrm{~S}$ & $57.9 E$ & 25 & French patron of letters \\
\hline Riley & $14.0 \mathrm{~N}$ & $72.2 \mathrm{E}$ & 25 & English botanist \\
\hline Roxanna & $26.5 \mathrm{~N}$ & $334.6 \mathrm{E}$ & 9 & Persian first name \\
\hline Samintang & $39.0 \mathrm{~S}$ & $80.6 \mathrm{E}$ & 24 & Korean poetess \\
\hline Saskia & $28.6 S$ & $337.2 \mathrm{E}$ & 40 & Rembrandt's wife(Dutch) \\
\hline Scarpellini & $23.4 S$ & $34.4 E$ & 25 & Italian astronomer \\
\hline Simonenko & 26.95 & $97.3 \mathrm{E}$ & 35 & Soviet astronomer \\
\hline stein & $30.0 \mathrm{~s}$ & $345.5 \mathrm{E}$ & 24 & American writer \\
\hline Stuart & $30.8 \mathrm{~s}$ & $20.2 \mathrm{E}$ & 67 & Mary, Quen of Scots \\
\hline Vigier Lebrun & $17.3 \mathrm{~N}$ & $141.3 \mathrm{E}$ & 53 & French painter \\
\hline Von Siebold & $52.0 \mathrm{~s}$ & $36.7 \mathrm{E}$ & 36 & German physician \\
\hline wilder & $17.4 \mathrm{~N}$ & $122.4 \mathrm{E}$ & 35 & American author \\
\hline Woolf & $37.7 S$ & $27.1 \mathrm{E}$ & 25 & British writer \\
\hline Xantippe & $10.9 \mathrm{~S}$ & $11.7 \mathrm{E}$ & 41 & Greek wife of Socrates \\
\hline Xiao Hong & & $101.5 \mathrm{E}$ & 37 & Chines novelist \\
\hline zhu Shuzhen & & $356.6 \mathrm{E}$ & 32 & Chinese poetess \\
\hline \multicolumn{5}{|l|}{ CORONA } \\
\hline Beyla Corona & $25.0 \mathrm{~N}$ & $15.5 \mathrm{E}$ & 400 & Norse Earth goddess \\
\hline Eithinoha Corona & $57.5 s$ & $7.5 \mathrm{E}$ & 500 & Iroquois Earth goddess \\
\hline Gertjon Corona & $30.0 \mathrm{~s}$ & $276.0 \mathrm{E}$ & 250 & Teutonic fertility goddess \\
\hline Hervor Corona & $25.5 s$ & $269.0 \mathrm{E}$ & 250 & Norse fertility goddess \\
\hline Kamui-Huci Corona & $63.5 \mathrm{~s}$ & $322.5 E$ & 300 & Jap. (Ainu) Earth goddess \\
\hline Otygen Corona & $57.0 \mathrm{~s}$ & $30.5 \mathrm{E}$ & 400 & Mongolian Earth mother \\
\hline Quetzalpetlatl Coror & 68 & $354.5 \mathrm{E}$ & 400 & Aztec fertility goddess \\
\hline Rigatona Corona & & $278.5 \mathrm{E}$ & 300 & Celtic fertility goddess \\
\hline
\end{tabular}


VENUS ( Cont. )

\begin{tabular}{|c|c|c|c|c|}
\hline Name & Lat & Long & $\begin{array}{l}\text { Diam } \\
(\mathrm{km})\end{array}$ & Attribute \\
\hline \multicolumn{5}{|l|}{ FLUCTUS } \\
\hline Eriu Fluctus & $38.0 \mathrm{~S}$ & $1.0 \mathrm{E}$ & 1200 & Irish Earth mother \\
\hline Kaiwan Fluctus & $48.0 \mathrm{~S}$ & $1.5 \mathrm{E}$ & 1200 & Ethiopian Earth mother \\
\hline Mylitta Fluctus & $56.0 \mathrm{~S}$ & $353.5 \mathrm{E}$ & 1250 & Semitic mother goddess \\
\hline \multicolumn{5}{|l|}{ FOSSA } \\
\hline Enyo Fossae & $63.0 \mathrm{~S}$ & $347.0 \mathrm{E}$ & 900 & Greek war goddess \\
\hline Nike Fossae & $59.5 \mathrm{~s}$ & $339.5 \mathrm{E}$ & 850 & Greek goddess of victory \\
\hline \multicolumn{5}{|l|}{ LINEA } \\
\hline Kalaipahoa Linea & $60.5 \mathrm{~S}$ & $336.5 \mathrm{E}$ & 2400 & Hawaiian war goddess \\
\hline Morrigan Linea & $54.5 \mathrm{~S}$ & $311.0 \mathrm{E}$ & 3200 & Celtic war goddess \\
\hline Penardun Linea & $54.0 \mathrm{~S}$ & $344.0 \mathrm{E}$ & 975 & Celtic sky goddess \\
\hline \multicolumn{5}{|l|}{ MONS } \\
\hline Nepthys Mons & $33.0 \mathrm{~S}$ & $317.5 \mathrm{E}$ & 350 & Egyptian land goddess \\
\hline \multicolumn{5}{|l|}{ REGIO } \\
\hline Dione Regio & $31.5 \mathrm{~s}$ & $323.0 \mathrm{E}$ & 2300 & Greek Titaness \\
\hline Hyndla Regio & $24.0 \mathrm{~N}$ & $294.5 \mathrm{E}$ & 2300 & Norse wood Giantess \\
\hline
\end{tabular}

SATELLITES OF SATURN AND NEPTUNE

$\begin{array}{lllr}\begin{array}{l}\text { Temporary } \\ \text { designation }\end{array} & \begin{array}{l}\text { Permanent } \\ \text { designation }\end{array} & \text { Name } & \begin{array}{r}\text { Distance from planet's } \\ \text { center }(\mathrm{km})\end{array} \\ \text { 1981S13 } & \text { Saturn XVIII } & \text { Pan } & 133,600 \\ & & & \\ \text { 1989N6 } & \text { Neptune III } & \text { Naiad } & 48,230 \\ \text { 1989N5 } & \text { Neptune IV } & \text { Thalassa } & 50,070 \\ \text { 1989N3 } & \text { Neptune V } & \text { Despina } & 52,530 \\ \text { 1989N4 } & \text { Neptune VI } & \text { Galatea } & 61,950 \\ \text { 1989N2 } & \text { Neptune VII } & \text { Larissa } & 73,550 \\ \text { 1989N1 } & \text { Neptune VIII } & \text { Proteus } & 117,640\end{array}$

NEPTUNE RINGS AND RING ARCS

$\begin{array}{lll}\text { 1989N3R } & \text { Galle(N42) } & 41,900 \\ \text { 1989N2R } & \text { Leverrier(N53) } & 53,200 \\ \text { 1989N1R } & \text { Adams(N63) } & 62,900 \\ & & \\ \text { "Leading" ring arc } & \text { Liberte } & 62,900 \\ \text { "Equidistant" ring arc } & \text { Egalite } & 62,900 \\ \text { "Following" ring arc } & \text { Fraternite } & 62,900\end{array}$

